\title{
Sorafenib combined with gemcitabine in EGFR-TKI-resistant human lung cancer cells
}

\author{
$\mathrm{JING} \mathrm{LI}^{1}$, YUE-YIN PAN ${ }^{2}$ and YING ZHANG ${ }^{1}$ \\ ${ }^{1}$ Department of Geriatrics, The Third Affiliated Hospital of Anhui Medical University, Hefei, Anhui 230061;
${ }^{2}$ Department of Oncology, The First Affiliated Hospital of Anhui Medical University, Hefei, Anhui 230022, P.R. China
}

Received July 17, 2012; Accepted September 26, 2012

DOI: $10.3892 / \mathrm{ol} .2012 .958$

\begin{abstract}
Sorafenib is a multi-targeted agent and has been reported to have potent antitumor effects against various types of tumors, including human non-small cell lung cancer (NSCLC). In this study, we explored in vitro the antitumor effects of sorafenib alone and in combination with gemcitabine in epidermal growth factor receptor-tyrosine kinase inhibitor (EGFR-TKI)-resistant human lung cancer cell lines and the related molecular mechanisms. The NSCLC cell lines A549 (mutant KRAS), H1666 (mutant BRAF) and H1975 (mutant EGFR-T790M) were treated with sorafenib and gemcitabine alone and in combination. The cytotoxicity was assessed by MTT assay, cell cycle distribution was analyzed by flow cytometry, and alterations in signaling pathways were analyzed by western blotting. We found that sorafenib exhibited dose-dependent growth inhibition in all three EGFR-TKI-resistant NSCLC cell lines. When sorafenib was combined with gemcitabine, synergistic activity was observed in the A549 and H1666 cells and antagonistic activity was observed in the H1975 cells. Sorafenib arrested the cell cycle at the G1 phase, whereas gemcitabine arrested the cell cycle at the $\mathrm{S}$ phase. Sorafenib inhibited C-RAF and p-ERK in the A549 cells and B-RAF and p-ERK in the H1666 and H1975 cells. The molecular mechanism of this synergism is that RAF/MEK/ERK which are activated by gemcitabine are efficiently suppressed by simultaneously administered sorafenib. By contrast, the mechanism of antagonism may be due to mutual interference with the cell cycle in the H1975 cells. In conclusion, we found that sorafenib exhibits antiproliferative effects in EGFR-TKI-resistant NSCLC cell
\end{abstract}

Correspondence to: Professor Ying Zhang, Department of Geriatrics, The Third Affiliated Hospital of Anhui Medical University, 390 Huai He Road, Hefei 230061, P.R. China

E-mail: zhangying19650108@163.com

Professor Yue-Yin Pan, Department of Oncology, The First Affiliated Hospital of Anhui Medical University, Hefei, Anhui 230022, P.R. China

E-mail: panyueyin@gmail.com

Key words: sorafenib, gemcitabine, non-small cell lung cancer, epidermal growth factor-tyrosine kinase inhibitor-resistance lines and when combined with gemcitabine demonstrates synergistic activity in A549 and H1666 cells but antagonistic activity in $\mathrm{H} 1975$ cells.

\section{Introduction}

Lung cancer is the leading cause of cancer-related mortality worldwide, approximately $80 \%$ of which is non-small cell lung cancer (NSCLC) (1). Currently, the traditional cytotoxic chemotherapy has reached a plateau with respect to efficacy, with limited survival benefits for advanced NSCLC patients.

Target-based therapies have improved survival rates in patients with a variety of cancers, including NSCLC. Certain epidermal growth factor receptor tyrosine kinase inhibitors (EGFR-TKIs), including gefitinib and erlotinib, have been approved as second-line treatments for lung cancer. However, EGFR-TKIs have little effect in NSCLC patients with KRAS, BRAF or EGFR-T790M mutations (2-4). The KRAS and BRAF mutations are tandem activating mutations in the RAS/RAF/MEK/ERK signaling pathway. Taken together, the KRAS or BRAF mutations may be present in approximately 50\% of NSCLCs (5). The EGFR-T790M mutation is associated with acquired resistance to EGFR-TKIs. Patients with this mutation are considered to be resistant to cytotoxic chemotherapeutic agents and unsuitable for EGFR-TKI treatment (6). Therefore, there is an urgent requirement to identify new treatment strategies to improve the clinical efficacy of NSCLC therapy.

Sorafenib (BAY 43-9006) is an oral multi-kinase inhibitor that targets tumor growth, survival and angiogenesis. Sorafenib inhibits tumor cell growth directly by affecting Raf kinases, which are critical proliferation and cell survival signaling molecules downstream of RAS in the RAF/MEK/ERK signaling pathway. It also inhibits tumor angiogenesis and metastasis by decreasing the activity of the vascular endothelial growth factor receptor-2/3 (VEGFR-2 and VEGFR-3) and platelet-derived growth factor receptor- $\beta$ (PDGFR- $\beta$ ) (7). Studies have shown that sorafenib inhibits MEK and ERK phosphorylation and has a wide range of antitumor effects in various cancer cell lines and tumor xenografts (8). Single-agent sorafenib has demonstrated clinical activity against NSCLC in the first-line setting (9-11).

Currently, there is interest in assessing the efficacy of sorafenib given in combination with conventional cytotoxic chemotherapy agents. Gemcitabine is a pyrimidine nucleoside 
antimetabolite agent with a favorable toxicity profile, which is active against a variety of human malignancies, including NSCLC, and has been frequently used in combinatorial treatments with other anticancer agents (12).

In the current study we examined in vitro the antitumor effect and related mechanisms of sorafenib combined with gemcitabine in EGFR-TKI-resistant NSCLC cell lines.

\section{Materials and methods}

Cell lines and chemicals. EGFR-TKI-resistant lung cancer cell lines A549 (mutant KRAS/wild-type EGFR), H1666 (mutant BRAF/wild-type EGFR) and H1975 (mutant EGFR-T790M/wild-type KRAS) were purchased from the American Type Culture Collection (Manassas, VA, USA) and were maintained in RPMI-1640 medium (Hyclone, Logan, UT, USA) with $10 \%$ heat-inactivated fetal bovine serum (Hyclone), $100 \mathrm{U} / \mathrm{ml}$ penicillin and $100 \mathrm{mg} / 1$ streptomycin at $37^{\circ} \mathrm{C}$ in a $5 \% \mathrm{CO}_{2}$ atmosphere. Sorafenib was obtained from Bayer (Leverkusen, Germany) and gemcitabine was purchased as a commercial product from our hospital pharmacy.

Analysis of the antitumor effects of sorafenib and gemcitabine. The MTT assay was used to determine the antitumor effects of each drug. Briefly, cells in 96-well plates were treated with escalating doses of sorafenib $(0.78-25 \mu \mathrm{M})$ or gemcitabine $(0.78-25 \mathrm{nM})$ for $72 \mathrm{~h}$ and then $20 \mu \mathrm{l}$ MTT solution was added to $200 \mu \mathrm{l}$ media in each well. The absorbance was determined at $490 \mathrm{~nm}$ after $4 \mathrm{~h}$ of incubation. The percentage of cell growth inhibition resulting from each drug was calculated as: $\left(\mathrm{OD}_{490 \text { control cells }}-\mathrm{OD}_{490 \text { treated cells }}\right) / \mathrm{OD}_{490 \text { control cells }} \mathrm{x} 100$. This assay was repeated as more than three independent experiments.

Determination of a synergistic antitumor effect between sorafenib and gemcitabine. The A549, H1666 and H1975 cells were concurrently exposed to sorafenib and gemcitabine for $72 \mathrm{~h}$. The combination drug dosing was carried out using constant ratios of the $\mathrm{IC}_{50}$ values. Thus, the combination index (CI) value was calculated using $0.125,0.25,0.5,1,2$ and 4 times the $\mathrm{IC}_{50}$ values of sorafenib and gemcitabine combination doses. The CI values of the interactions between sorafenib and gemcitabine were analyzed according to the Chou and Talalay method using CompuSyn software (ComboSyn, Inc., Paramus, NJ, USA). $\mathrm{CI}>1, \mathrm{CI}=1$ and $\mathrm{CI}<1$ indicated antagonistic, additive and synergistic effects, respectively (13).

Cell cycle analysis. Cell cycle analysis was conducted using flow cytometry. The A549, H1666 and H1975 cells were treated with sorafenib and gemcitabine as single agents or in combination using constant ratios of the $\mathrm{IC}_{50}$ levels, harvested after $72 \mathrm{~h}$ and fixed in $80 \%$ ethanol on ice. All cell samples were kept in a $-2^{\circ} \mathrm{C}$ freezer until analysis by flow cytometry. Once the cells were removed from the freezer, $1 \mathrm{ml}$ cold phosphate-buffered saline (PBS) was added and the samples were centrifuged. After washing with cold PBS twice, DNA staining was performed using a solution of propidium iodide $(0.05 \mathrm{mg} / \mathrm{ml})$ and RNase $(2 \mathrm{mg} / \mathrm{ml})$ for $30 \mathrm{~min}$ at room temperature. Cells were analyzed using a FACScan cytometer and the percentages of cells in the G1, S and G2/M phases of the cell cycle were estimated using Cell Lab Quanta SC Software.
Table I. $\mathrm{IC}_{50}$ values of sorafenib and gemcitabine determined by MTT assay.

\begin{tabular}{lcrr}
\hline $\mathrm{IC}_{50}$ & $\mathrm{~A} 549$ & $\mathrm{H} 1666$ & $\mathrm{H} 1975$ \\
\hline Sorafenib $(\mu \mathrm{M})$ & $4.08 \pm 0.14$ & $5.52 \pm 0.53$ & $2.54 \pm 0.01$ \\
Gemcitabine $(\mathrm{nM})$ & $9.21 \pm 0.36$ & $11.65 \pm 0.19$ & $6.73 \pm 0.11$ \\
\hline
\end{tabular}

Western blot analysis. A549, H1666 and H1975 cells (1x10\% well) were treated with sorafenib and gemcitabine as single agents and in combination for $72 \mathrm{~h}$. The cells were washed with ice-cold PBS solution and scraped in lysis buffer. The lysates were centrifuged at $14,000 \mathrm{rpm}$ for $30 \mathrm{~min}$ at $4^{\circ} \mathrm{C}$ and the supernatant was collected. Equivalent amounts of proteins were analyzed by sodium dodecyl sulfate-polyacrylamide gel electrophoresis (SDS-PAGE) and transferred to PVDF membranes. Appropriate primary antibodies to B-RAF, C-RAF, pAKT, AKT, pERK1/2, ERK1/2, Bcl-2 and $\beta$-actin, purchased from Cell Signaling Technology (Beverly, MA, USA), were used. Proteins were visualized with a horseradish peroxidase-coupled secondary antibody from Cell Signaling Technology. Specific bands were detected using an enhanced chemiluminescence reagent (ECL; PerkinElmer Life Sciences, Inc., Boston, MA, USA) on autoradiographic film and quantitated by densitometry.

Statistical analysis. Unless specified, the results are expressed as the mean \pm SD of data collected from at least three independent experiments. The Student's t-test was used to determine the differences between control and treatment groups. $\mathrm{P}<0.05$ was considered to indicate a statistically significant result.

\section{Results}

Antiproliferative effects of sorafenib and gemcitabine alone or in combination in EGFR-TKI-resistant NSCLC cells. Sorafenib and gemcitabine showed a dose- and time-dependent antitumor effect in the A549, H1666 and H1975 cells (Fig. 1). After 72-h exposure to sorafenib, the $\mathrm{IC}_{50}$ values in the A549, H1666 and H1975 cells were 4.08 $\pm 0.14,5.52 \pm 0.53$ and $2.54 \pm 0.01 \mu \mathrm{M}$, respectively. The $\mathrm{IC}_{50}$ values of sorafenib in the three cells are within the clinically relevant concentration range for this drug (14). We conclude that the EGFR-TKI-resistant NSCLC cells are sensitive to sorafenib. Table I summarizes the $\mathrm{IC}_{50}$ values of gemcitabine and sorafenib.

When exposed to a combination of sorafenib and gemcitabine, synergistic activity was observed in the A549 and H1666 cells $(\mathrm{CI}<1)$, while antagonistic activity was observed in the H1975 cells (CI>1; Fig. 2).

Cell cycle effects of sorafenib and gemcitabine. Flow cytometry was used to evaluate the cell cycle phase distributions in the NSCLC cells following single-drug and concurrent administration of gemcitabine and sorafenib for $72 \mathrm{~h}$. Following sorafenib treatment, the proportions of the A549, H1666 and $\mathrm{H} 1975$ cells in the G0/G1 phase were increased, relative to control values $(\mathrm{P}<0.05)$. With gemcitabine alone, the $\mathrm{S}$ phase fraction increased in the three cell lines $(\mathrm{P}<0.05$; Fig. 3$)$. 
A

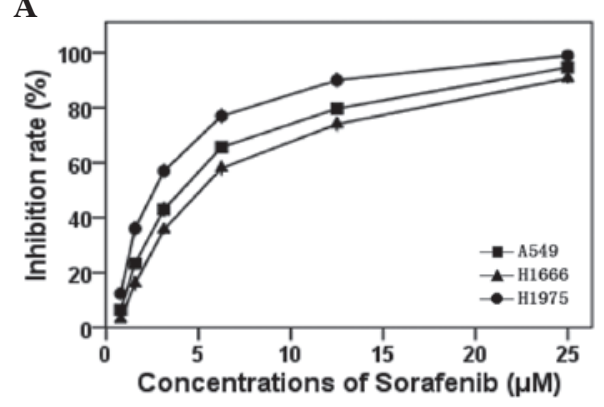

B

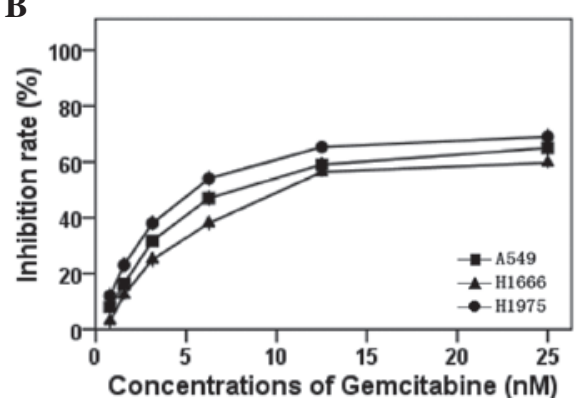

Figure 1. MTT assays were used to examine the inhibitory activities of sorafenib and gemcitabine on cell proliferation. The cells were exposed to various concentrations of (A) sorafenib (0.78-25 $\mu \mathrm{mol} / \mathrm{l})$ and (B) gemcitabine (0.78-25 nmol/1) in A549, H1666 and H1975 cells for $72 \mathrm{~h}$. Each data point is the result of more than three independent experiments.

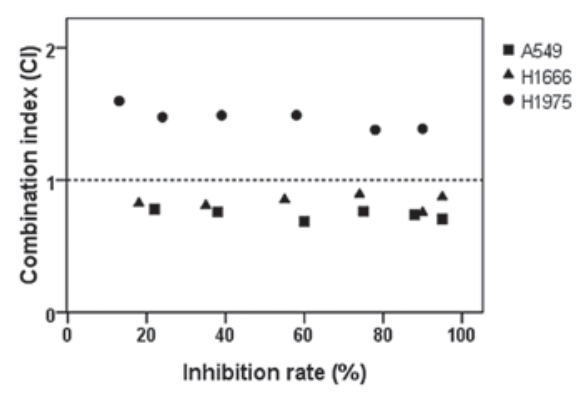

Figure 2. The combination index (CI) value of sorafenib combined with gemcitabine in A549, H1666 and H1975 cells was calculated using the Chou-Talalay method. $\mathrm{CI}<1$ in the A549 and H1666 cell lines; CI $>1$ in the H1975 cell line.

When exposed to the combination of gemcitabine and sorafenib, the proportions of cells in the G0/G1 and S phases increased in the A549 and $\mathrm{H} 1666$ cells $(\mathrm{P}<0.05)$. However, in the H1975 cells, the proportion of cells in the G0/G1 phase increased and the proportion in the $\mathrm{S}$ phase decreased (Fig. 3).

Effects of sorafenib and gemcitabine alone or in combination on downstream signaling pathways. We identified that gemcitabine upregulated the level of p-ERK in the three cell lines and increased the level of p-AKT in the H1975 cells at its $\mathrm{IC}_{50}$. When the A549 and H1666 cells were exposed to sorafenib at its $\mathrm{IC}_{50}$ for $72 \mathrm{~h}$, the levels of p-AKT, p-ERK and $\mathrm{Bcl}-2$ were decreased compared with those of unexposed cells, while in H1975 cells, sorafenib decreased only the levels of p-AKT (Fig. 4). We found that sorafenib inhibited C-RAF in A549 cells but B-RAF in H1666 and H1975 cells (Fig. 5).

In addition, we identified that when the A549 and H1666 cells were exposed to a combination of gemcitabine and sorafenib for $72 \mathrm{~h}$, the levels of p-AKT, p-ERK and Bcl-2 were decreased compared with their levels in the control, whereas, in the H1975 cells, the combination of the two drugs increased the levels of p-AKT and p-ERK. However, there was no significant variation in the total ERK and AKT expression levels compared with the control (Fig. 4).

\section{Discussion}

Targeted anticancer drugs, including the EGFR-TKIs gefitinib and erlotinib, have been approved for the treatment of lung
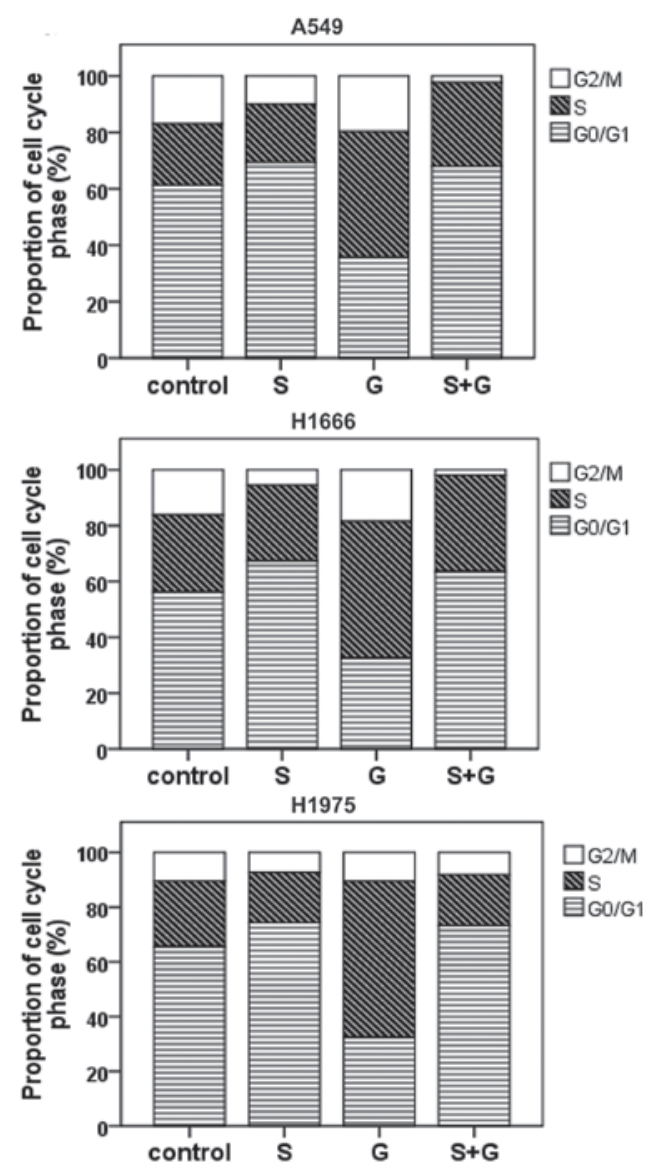

Figure 3. Flow cytometric analysis was used to determine the alterations in the cell cycle distributions in non-small cell lung cancer (NSCLC) cell lines following the administration of gemcitabine and sorafenib as single-agents or concurrently for $72 \mathrm{~h}$. The concentrations of gemcitabine and sorafenib used were at $\mathrm{IC}_{50}$ levels. $\mathrm{G}$, gemcitabine; $\mathrm{S}$, sorafenib; $\mathrm{S}+\mathrm{G}$, concurrent administration of sorafenib and gemcitabine.

cancer. Patients with EGFR mutations may greatly benefit from treatment with EGFR-TKIs (15-17). However, the presence of KRAS, BRAF and EGFR-T790M mutations are associated with primary or acquired resistance to EGFR-TKIs. The development of new treatment strategies for NSCLC patients is thus an important clinical goal.

There is multilevel cross-stimulation among the targets in lung cancer; when only one of these pathways is blocked others act as salvage or escape mechanisms for the cancer 

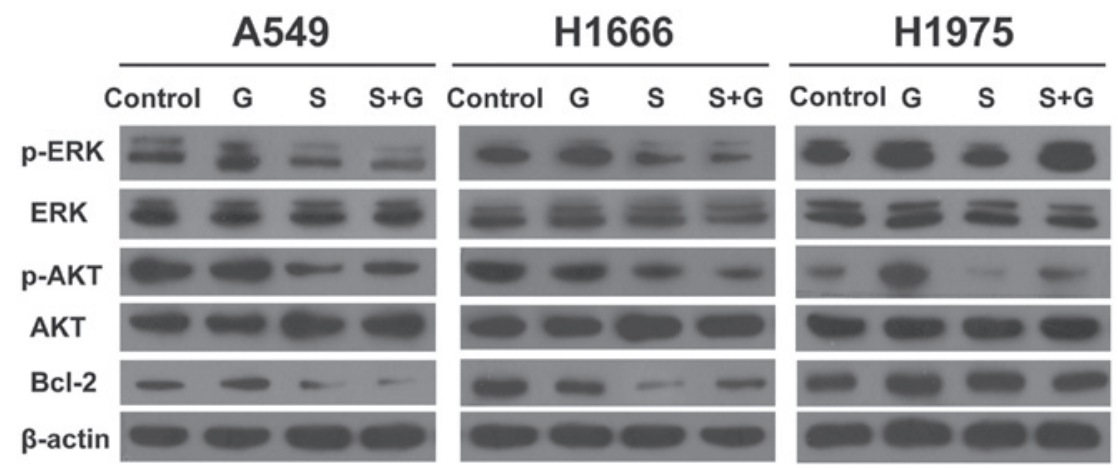

Figure 4. The effects of sorafenib and gemcitabine alone and in combination for $72 \mathrm{~h}$ on the expression levels of downstream signaling pathways in A549, H1666 and $\mathrm{H} 1975$ cells were evaluated by western blot analysis. G, gemcitabine; S, sorafenib; S+G, concurrent administration of sorafenib and gemcitabine.

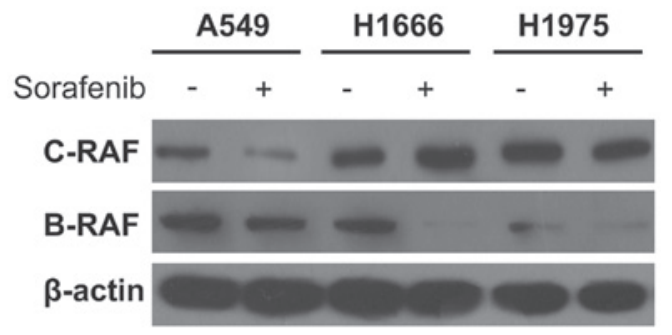

Figure 5. Effects of sorafenib on the expression levels of B-RAF and C-RAF in A549, H1666 and H1975 cells evaluated by western blot analysis.

cells. Anticancer agents that interfere at different stages may preclude an escape or salvage mechanism for the cancer cells and therefore be more effective than single-targeted agents (18). The RAS/RAF/MEK/ERK and RAS/PI3K/PTEN/AKT pathways interact with each other to regulate growth and play key roles in the transmission of proliferative signals, in some cases contributing to tumorigenesis. In NSCLC, the activation of KRAS and BRAF leads to ERK1/2 overexpression through the RAF/MEK/ERK signaling pathway (19-21). Hence, inhibition of the RAS/RAF/MEK/ERK signaling pathway is an important strategy in anticancer drug development. Sorafenib is a novel, multi-kinase inhibitor that targets tumor proliferation and tumor angiogenesis (7). It has been approved for the treatment of advanced renal cell cancer (RCC) and is currently being evaluated for the treatment of other tumors.

The present study was performed in EGFR-TKI-resistant A549 (mutant KRAS/wild-type EGFR), H1666 (mutant BRAF/wild-type EGFR) and H1975 (mutant EGFR-T790M/ wild-type KRAS) human lung cancer cell lines to investigate the antiproliferative effects of sorafinib as a single agent and in combination with gemcitabine. We found that sorafenib and gemcitabine exhibited dose dependent growth inhibition of cell growth when used as single agents to treat A549, H1666 and H1975 lung cancer cells. This suggests that sorafenib is efficacious for inhibiting growth in EGFR-TKI-resistant NSCLC cell lines. Synergism was observed when sorafenib was combined with gemcitabine in the A549 and H1666 cells, whereas antagonistic interactions were observed in the H1975 cells.

The synergistic and antagonistic effects may be explained by differences in the changes induced in the cell cycle. In our study, sorafenib arrested cells at the G1 phase and gemcitabine caused S phase accumulation in all three cell lines. When exposed to a combination of gemcitabine and sorafenib, the proportions of cells in the G0/G1 and S phases increased in the A549 and H1666 cells. We conclude that sorafenib and gemcitabine modulate the cell cycle phase independently of each other in A549 and H1666 cells and generated an additive effect, without disturbing each other. However, in the H1975 cells, the proportion of cells in the G0/G1 phase increased and in the $\mathrm{S}$ phase decreased. Sorafenib mainly arrested cells at the G1 phase, thereby interfering with the cytotoxic effects of gemcitabine in the $\mathrm{S}$ phase, resulting in decreased cytotoxicity.

The differences in the antiproliferative effects of sorafenib combined with gemcitabine may also result from their effects on growth signaling pathways. We identified that gemcitabine increased the levels of p-ERK in the A549, H1666 and H1975 cells and in the H1975 cells, p-AKT levels were also upregulated by gemcitabine. Similar to our results, a previous study reported that cell signaling pathways may be gradually activated by chemotherapy (22). Phosphorylated ERK and AKT play important roles in tumor cell proliferation; gemcitabine induced ERK and AKT phosphorylation, leading to the prevention of apoptosis.

We have shown that sorafenib inhibited the activity of p-AKT, p-ERK and Bcl-2 in the A549 and H1666 cells after $72 \mathrm{~h}$ exposure, while in the H1975 cells, sorafenib downregulated the level of p-AKT but not the levels of p-ERK and Bcl-2. We conclude that sorafenib inhibited the expression of Bcl-2 in the A549 and H1666 cells, mainly due to its inhibition of the RAF/MEK/ERK downstream pathway. We also identified that sorafenib decreased the level of C-RAF in the A549 cells and of B-RAF in the H1666 and H1975 cells. These results are similar to those in a previous study which reported that sorafenib inhibits NSCLC cell growth by targeting B-RAF in KRAS wild-type cells and C-RAF in KRAS mutant cells. However, this previous study demonstrated that sorafenib failed to inhibit p-ERK in NSCLC cell lines with KRAS mutations (23). The different results may be attributed to a shorter time of exposure to sorafenib.

When sorafenib was combined with gemcitabine, a significant decrease in p-AKT, p-ERK and Bcl-2 levels was observed in the A549 and H1666 cells, as compared with the control. We conclude that the RAF/MEK/ERK signaling pathways activated by gemcitabine may be blocked by sorafenib in 
KRAS or BRAF mutant cells. This may be due to sorafenib inhibiting mutant RAS and RAF kinases and directly blocking the RAF/MEK/ERK pathway. However, the levels of p-AKT and p-ERK increased when sorafenib and gemcitabine were applied together to the H1975 cells, demonstrating that the downstream pathway activated by gemcitabine was not blocked by sorafenib in cells harboring a EGFR-T790M mutation. These observations of p-AKT, p-ERK and Bcl-2 in NSCLC cells may explain the synergistic or antagonist growth inhibitory effects observed in the three cell lines treated with sorafenib and gemcitabine.

In conclusion, we found that sorafenib exhibited significant growth inhibitory effects in EGFR-TKI-resistant NSCLC cells. Regardless of the mutation status of KRAS, BRAF or EGFR T790M, sorafenib was a treatment of choice against NSCLC. Moreover, sorafenib combined with gemcitabine generated synergistic effects in KRAS or BRAF mutant cells, and antagonistic effects in EGFR-T790M mutant cells. These data encourage the development of sorafenib as a single targeted therapy or in combination with cytotoxic chemotherapy drugs for treatment of NSCLC.

\section{Acknowledgements}

This study was supported by a grant from the Anhui Provincial Science and Technology Agency Foundation of China (No. 09020303042) and supported by The Central Laboratory of The Third Affiliated Hospital of Anhui Medical University.

\section{References}

1. Jemal A, Siegel R, Xu J and Ward E: Cancer statistics, 2010. CA Cancer J Clin 60: 277-300, 2010.

2. Pao W, Wang TY, Riely GJ, et al: KRAS mutations and primary resistance of lung adenocarcinomas to gefitinib or erlotinib. PLoS Med 2: e17, 2005.

3. McCubrey JA, Steelman LS, Chappell WH, et al: Roles of the Raf/MEK/ERK pathway in cell growth, malignant transformation and drug resistance. Biochim Biophys Acta 1773: 1263-1284, 2007.

4. Gazdar AF: Activating and resistance mutations of EGFR in nonsmall-cell lung cancer: role in clinical response to EGFR tyrosine kinase inhibitors. Oncogene 28 (Suppl 1): S24-S31, 2009.

5. Adjei AA: K-ras as a Target for Lung Cancer Therapy. J Thorac Oncol 3 (Suppl 2): S160-S163, 2008.

6. Ladanyi $\mathrm{M}$ and Pao W: Lung adenocarcinoma: guiding EGFRtargeted therapy and beyond. Mod Pathol 21: S16-S22, 2008.

7. Wilhelm SM, Carter C, Tang L, et al: BAY 43-9006 exhibits broad spectrum oral antitumor activity and targets the $\mathrm{RAF} / \mathrm{MEK} / \mathrm{ERK}$ pathway and receptor tyrosine kinases involved in tumor progression and angiogenesis. Cancer Res 64: 7099-7109, 2004.
8. Carter CA, Chen C, Brink C, et al: Sorafenib is efficacious and tolerated in combination with cytotoxic or cytostatic agents in preclinical models of human non-small cell lung carcinoma. Cancer Chemother Pharmacol 59: 183-195, 2007.

9. Dal Lago L, D'Hondt V and Awada A: Selected combination therapy with sorafenib: a review of clinical data and perspectives in advanced solid tumors. Oncologist 13: 845-858, 2008.

10. Blumenschein GR Jr, Gatzemeier U, Fossella F, et al: Phase II, multicenter, uncontrolled trial of single-agent sorafenib in patients with relapsed or refractory, advanced non-small-cell lung cancer. J Clin Oncol 27: 4274-4280, 2009.

11. Dy GK, Hillman SL, Rowland KM Jr, et al: A front-line window of opportunity phase 2 study of sorafenib in patients with advanced nonsmall cell lung cancer: North Central Cancer Treatment Group Study N0326. Cancer 116: 5686-5693, 2010.

12. Dougherty DW and Friedberg JW: Gemcitabine and other new cytotoxic drugs: will any find their way into primary therapy? Curr Hematol Malig Rep 5: 148-156, 2010.

13. Chou TC and Talalay P: Quantitative analysis of dose-effect relationships: the combined effects of multiple drugs or enzyme inhibitors. Adv Enzyme Regul 22: 27-55, 1984.

14. Strumberg D, Clark JW, Awada A, et al: Safety, pharmacokinetics, and preliminary antitumor activity of sorafenib: a review of four phase I trials in patients with advanced refractory solid tumors. Oncologist 12: 426-437, 2007.

15. Mok TS, Wu YL, Thongprasert S, et al: Gefitinib or carboplatin-paclitaxel in pulmonary adenocarcinoma. N Engl J Med 361: 947-957, 2009.

16. Maemondo M, Inoue A, Kobayashi K, et al: Gefitinib or chemotherapy for non-small-cell lung cancer with mutated EGFR. N Engl J Med 362: 2380-2388, 2010.

17. Masago K, Fujita S, Togashi Y, et al: Clinicopathologic factors affecting the progression-free survival of patients with advanced non-small-cell lung cancer after gefitinib therapy. Clin Lung Cancer 12: 56-61, 2011

18. Petrelli A and Giordano S: From single- to multi-target drugs in cancer therapy: when aspecificity becomes an advantage. Curr Med Chem 15: 422-432, 2008.

19. Linardou H, Dahabreh IJ, Kanaloupiti D, et al: Assessment of somatic K-Ras mutations as a mechanism associated with resistance to EGFR-targeted agents: a systematic review and meta-analysis of studies in advanced non-small cell lung cancer and colorectal cancer. Lancet Oncol 9: 962-972, 2008.

20. Lopez-Chavez A, Carter CA and Giaccone G: The role of KRAS mutations in resistance to EGFR inhibition in the treatment of cancer. Curr Opin Investig Drugs 10: 1305-1314, 2009.

21. Kotoula V, Sozopoulos E, Litsiou H, et al: Mutational analysis of the BRAF, RAS and EGFR genes in human adrenocortical carcinomas. Endocr Relat Cancer 16: 565-572, 2009.

22. Torres K and Horwitz SB: Mechanisms of taxol-induced cell death are concentration dependent. Cancer Res 58: 3620-3626, 1998.

23. Takezawa K, Okamoto I, Yonesaka K, Hatashita E, Yamada Y, Fukuoka $M$ and Nakagawa K: Sorafenib inhibits non-small cell lung cancer cell growth by targeting B-RAF in KRAS wild-type cells and C-RAF in KRAS mutant cells. Cancer Res. 69: 6515-6521, 2009. 